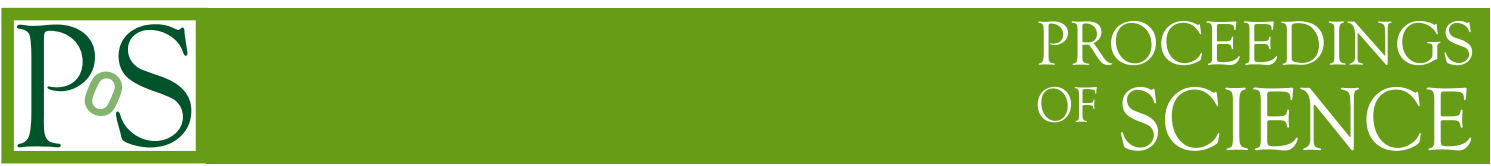

\title{
Measurements of Heavy Nuclei with the CALET Experiment
}

Yosui Akaike* for the CALET Collaboration

University of Maryland, Baltimore County and NASA Goddard Space Flight Center 8800 Greenbelt Rd. Greenbelt, MD 20771, USA

E-mail: yosui.akaike@nasa.gov

The CALorimetric Electron Telescope, CALET, was installed on the International Space Station in August 2015 and it has been collecting data since October 2015. Direct measurements of the high energy spectra of individual cosmic ray nuclei and of the energy dependence of secondary-toprimary abundance ratios are important for an understanding of the acceleration and propagation of cosmic rays in the galaxy. CALET is able to identify cosmic ray nuclei with individual element resolution and measure their energies in the range from a few tens of $\mathrm{GeV}$ to the PeV scale. The instrument consists of two layers of segmented plastic scintillators to measure the particle charge, from $\mathrm{Z}=1$ to 40 , a 3 radiation length thick tungsten-scintillating fiber imaging calorimeter, and a 27 radiation length thick PWO calorimeter. In this paper, the capability of charge identification and first results for heavy nuclei $(\mathrm{Z}>8)$ up to iron with CALET are presented.

35th International Cosmic Ray Conference - ICRC2017

10-20 July, 2017

Bexco, Busan, Korea

* Speaker. 


\section{Introduction}

Precise measurements of cosmic-ray fluxes are important to understand their origin and propagation in our Galaxy. While acceleration via supernova shock waves and diffusive propagation in the Galactic magnetic fields are commonly accepted as a feasible scenario for cosmic-ray origins, there are many outstanding questions about the details. Recent observations show that the spectra of protons and helium are hardened around several hundred $\mathrm{GeV}[1,2]$, which imply some unknown processes in the acceleration or propagation of cosmic rays neglected in the simple traditional scenario. Observed spectra of heavier nuclei also indicate hardening above $\sim 200 \mathrm{GeV} /$ nucleon [3]. Various models have been proposed to account for these spectral features, but the discussion cannot be concluded until more precise observations of each cosmic-ray component are carried out in a wider energy range.

CALET is a detector optimized to observe electrons and is also available to detect hadronic components up to the highest energy region ever directly observed. High and continuous energy resolution provided by a thick calorimeter is especially important for revealing the spectral features of each element. Reliable charge and track identification based on segmented scintillator paddles and scintillating fiber ribbons is also a major advantage of CALET. These capabilities for the measurement of nuclei have been verified in several beam tests [4-7]. The detector was installed on the International Space Station in August 2015 and has been collecting data since October 2015. In this paper, we report the first preliminary results of heavy cosmic-ray nuclei $Z>8$ with the data obtained in the period of October 132015 to March 31 2017. The results of electrons [8], light nuclei $(Z \leq 8)$ [9] and ultra heavy nuclei [10] are also reported in this conference volume.

\section{Calorimeter}

The CALET detector is composed of three main detector subsystems. The top part (CHarge Detector; CHD) consists of 2 layers of 14 segmented plastic scintillators, each of which has dimensions of $3.2 \mathrm{~cm} \times 45.0 \mathrm{~cm} \times 1.0 \mathrm{~cm}$. The CHD provides the primary charge determination for incident particles. The middle part (IMaging Calorimeter; IMC) is composed of 7 tungsten plates and 8 detection layers made of scintillating fibers (SciFis). Each layer consists of two SciFi belts arranged in the $x$ and $y$ directions. Each SciFi belt is made of 448 SciFis with a $1 \mathrm{~mm}$ square cross section and $448 \mathrm{~mm}$ in length. The tungsten plates interleaved between the SciFi layers have thicknesses of $0.2 X_{0} \times 5$ layers and $1.0 X_{0} \times 2$ layers. The IMC is mainly used for track reconstruction and is also useful for charge identification. The bottom part of the detector (Total AbSorption Calorimeter; TASC) is composed of 12 detection layers. Each layer has 16 PWO logs; each log has dimensions $1.9 \mathrm{~cm} \times 2.0 \mathrm{~cm} \times 32.6 \mathrm{~cm}$. The layers are arranged in alternating orthogonal directions to provide a 3-dimensional image of the shower development. The total thickness of the calorimeter is 30 radiation length $\left(\mathrm{X}_{0}\right)$ for electromagnetic particles and 1.3 interaction length $\lambda$ for protons.

Several beam tests carried out at the CERN-SPS verified the detector's capabilities, and validated the Monte Carlo simulation. Detector capabilities, such as energy resolution, angular resolution and electron/proton separation power for $10-290 \mathrm{GeV}$ electrons and $30-400 \mathrm{GeV}$ protons were 
studied in detail $[4,5]$. Charge and energy measurements for nuclei with the energies of 30 and 150 $\mathrm{GeV} / \mathrm{c}$ were also found to be consistent with predictions from Monte Carlo simulations $[6,7]$.
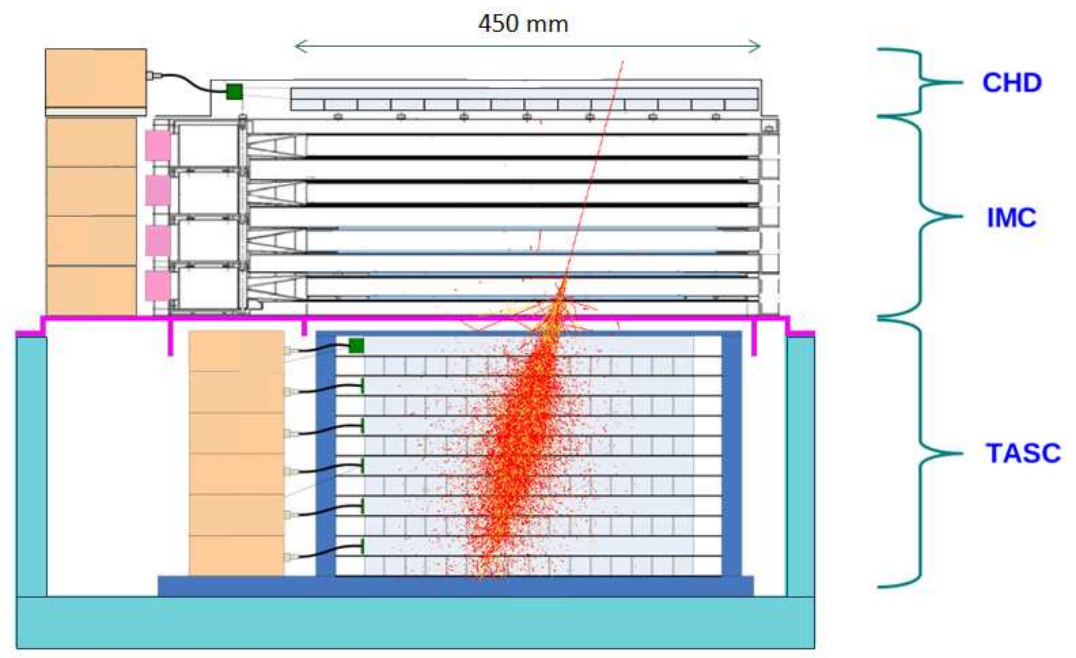

Figure 1: Side view of the CALET detector with an example of a simulated $1 \mathrm{TeV}$ electron shower image.

\section{Data Analysis}

On-board trigger for heavy nuclei Heavy ions are detected by the High Energy shower trigger (HE trigger), which is a principal trigger mode of the CALET observations [11]. The energy thresholds of the HE trigger at the bottom two layers of the IMC and the top layer of the TASC are set to detect shower events with energies over $10 \mathrm{GeV}$. While penetrating light nuclei like protons and helium are not triggered in this mode, heavy ions with $Z>8$ that interact in deep layers are detected because the $\mathrm{d} E / \mathrm{d} x$, which is proportional to $Z^{2}$, is large enough to exceed the trigger threshold. The trigger efficiency for ions with $Z>8$ is therefore almost $100 \%$. Figure 2 shows an example of an iron event with $9.3 \mathrm{TeV}$ in shower energy in the flight data.

Calibration and detector response Energy calibration for each channel has been carried out using penetrating cosmic-ray protons and helium [12]. The gain of each channel was equalized with this MIP calibration method. The position, temperature and time dependence of signals from all plastic scintillators and PWO logs have been well studied and calibrated. The scintillation quenching effect in the CHD, which reduces the light yields of highly ionizing particles and causes non-linearity between detector response and deposited energy, was analyzed based on the halo model [13] described by:

$$
\frac{d L}{d x}=A\left(\frac{1-f}{1+B(1-f) \frac{d E}{d x}}+f\right) \frac{d E}{d x}
$$

where $\mathrm{d} L / \mathrm{d} x$ is the specific scintillation light yield, $\mathrm{d} E / \mathrm{d} x$ is the rate of energy loss with path length, $f$ is the fraction of energy loss in the halo region, $B$ is a quenching parameter related to the strength 

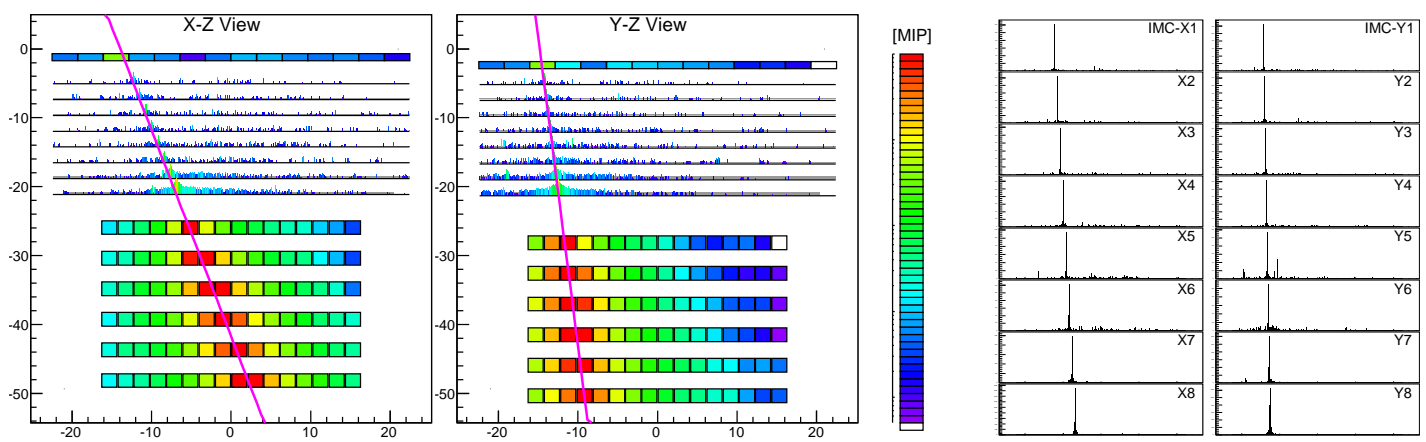

Figure 2: An example of an iron event which interact in the IMC with a $9.3 \mathrm{TeV}$ shower energy. The left figure shows the shower images with a reconstructed track (purple line) for $\mathrm{X}-\mathrm{Z}$ view and Y-Z view. The right figure shows the pulse height of each of the IMC layers in linear scale of MIP.

of the saturation and $A$ is a normalization constant. Figure 3 shows the peak values of the signal distributions of each charge derived from the flight data as a function of $Z^{2}$ with the fitting function based on Eq. 3.1. The parameters obtained from the fit were implemented in the Monte Carlo simulations to evaluate charge selection efficiencies.

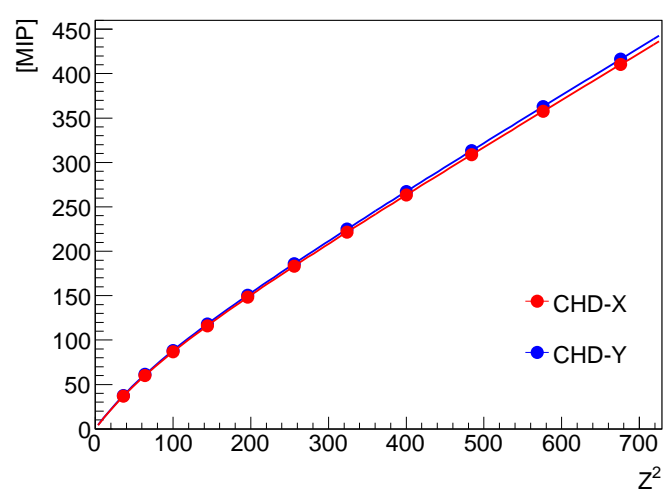

Figure 3: Peak value of CHD signals of each charge as a function of $Z^{2}$

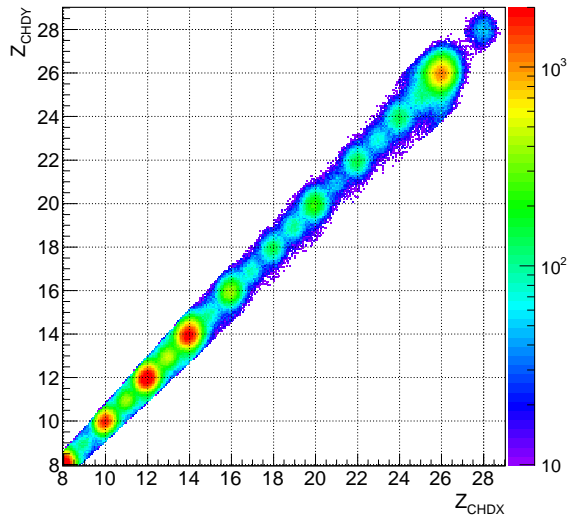

Figure 4: Correlation between charges from CHD-X and CHD-Y

Track reconstruction Reconstruction of the shower axis and estimation of the impact point at the CHD are essential for the charge determination of heavy nuclei. Although heavy ions can create many shower particles in the IMC, which could be a large background for track reconstruction, the signal of the primary particle is commonly larger than the signals of the shower particles (downward and back-scattered) as shown in Fig. 2, thanks to its large $\mathrm{d} E / \mathrm{d} x$. The track is therefore able to be reconstructed by a least-square fit in the upper four IMC layers. The tracks of heavy ions with $Z>8$ that interact in the 5 th IMC layer or deeper can be sufficiently reconstructed by this way. The impact point at the CHD can be estimated with an accuracy $330 \mu \mathrm{m}$ with CHD-X and $300 \mu \mathrm{m}$ with CHD-Y.

Charge consistency cuts and charge identification To maintain good charge resolution and 
remove events that interact in the $\mathrm{CHD}$, we require consistency in the $\mathrm{CHD}$ and IMC layers before estimating the charge of each event. Figure 4 shows the correlation between the charge estimated from CHD-X and CHD-Y. Events where the difference of CHD-X and CHD-Y is less than 15\% are selected. Consistency of the charge average using the 1st and 2nd $(x, y)$ IMC layers to the average obtained using 3rd and 4th $(x, y)$ IMC layers is also required. Applying these cuts rejects events with poor charge resolution due to interactions in the upper part of the detector or because of poor track reconstruction, allowing only events that interact in the 5th layer of the IMC or deeper. Efficiency of these cuts is $65-70 \%$ for heavy nuclei $(Z>8)$ with little energy dependence.

Charge determinations in CALET are primarily based on the signals from the CHD paddles on the reconstructed tracks. Figure 5 is the distribution of estimated charge from $Z=5$ (boron) to $\mathrm{Z}=28$ (nickel). Events within \pm 0.5 charge units of the peak are identified as particular elements in the flux analysis presented in this paper.

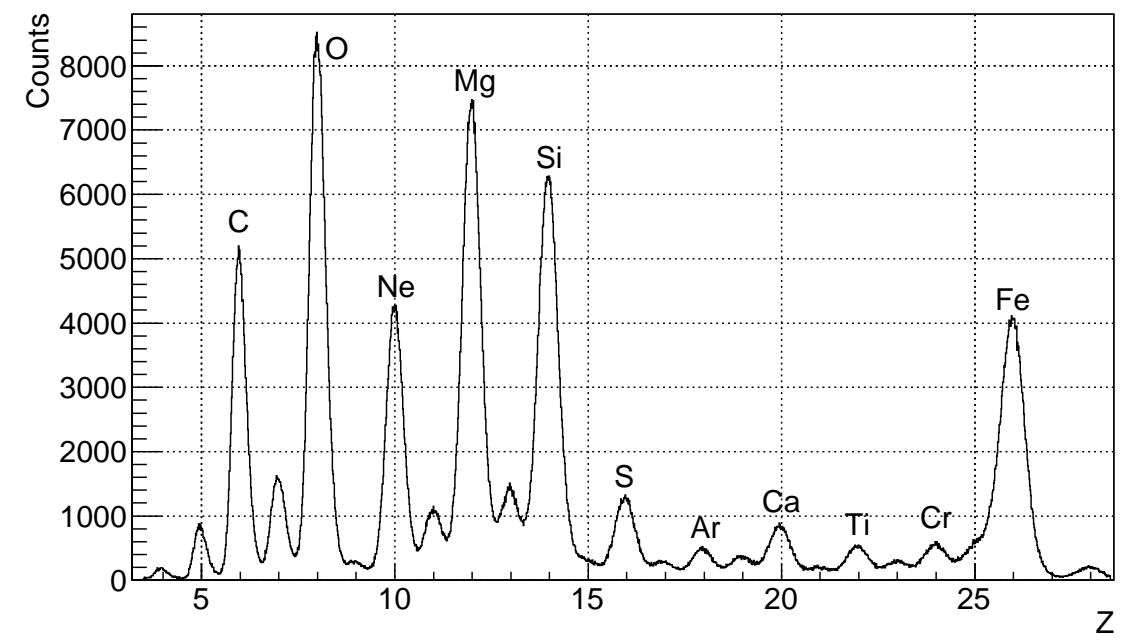

Figure 5: Distribution of estimated charge after consistency cuts using CHD and IMC.

Energy measurement and unfolding The shower energy of each event is determined from the sum of the TASC signals. The left top plot in Fig. 6 shows the distribution of the deposit energy in TASC of iron. Since energy leakage from the calorimeter is unavoidable due to the characteristics of hadron induced showers and the finite detector resolution, we applied an unfolding procedure for the derivation of the primary energy spectrum, based on Bayes's theorem [14] with the RooUnfold package [15]. Response functions were made from detailed Monte Carlo simulations using EPICS9.21 and Cosmos8.01 [16] with DPMJET-III [17] for the hadron interaction model. These are the same simulation methods the reliability of which was verified in several beam tests at the CERN-SPS [4,7]. The simulated events were generated isotropically at a rate of $\mathrm{d} N / \mathrm{d} E \propto E^{-1}$, and each was weighted with a factor to reproduce the desired power law spectrum. Instrumental properties such as pedestal RMS noise, photostatistics variation, quenching and saturation effects were implemented in the instrument response function. The same analysis procedures used in the flight data were applied to the simulation data. 
Selection efficiencies of the charge consistency cuts and charge identification method were taken into account in the unfolding process. Contaminants from the neighboring charged nuclei were also deconvolved. The left bottom plot in Fig. 6 shows the contaminant ratio from neighboring charged nuclei to iron estimated from MC simulations. The spectral indices of the background were assumed to be the same as those for the target nuclei, and the absolute value of the background was normalized based on the abundance ratio which was obtained from the charge distribution of CHD derived from flight data. The right top plot in Fig. 6 shows the unfolded energy distribution of iron. The initial power law index in the unfolding procedure was set to -2.60 . In order to study the uncertainty in the unfolding procedure due to the initial spectral index used two other indices were tested: -2.50 and -2.70 . In this index range, the uncertainty is less than $2 \%$ as shown in the right bottom plot in Fig. 6, which is significantly smaller than the statistical errors at high energies.
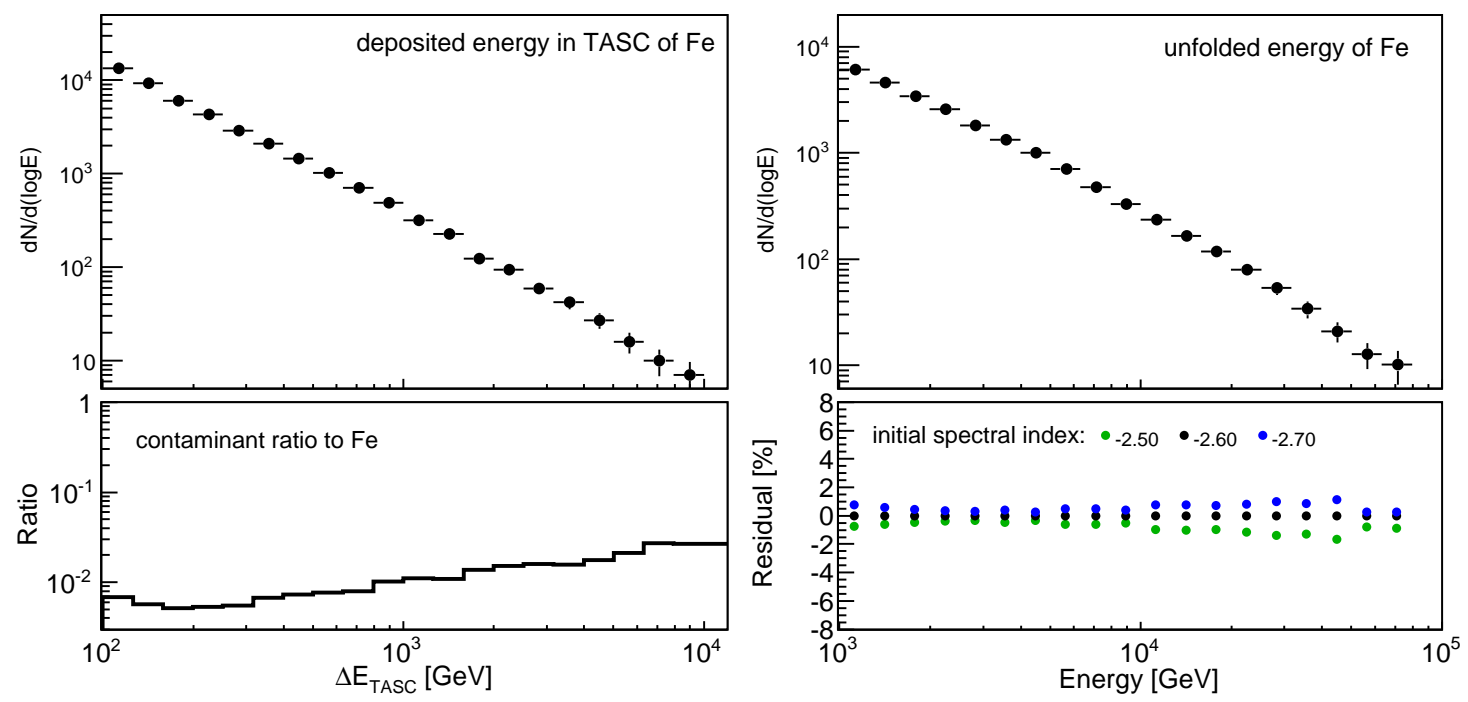

Figure 6: (Left top) The energy distribution in TASC of iron. (Left bottom) Contaminant ratio from neighboring charged nuclei to iron estimated from MC simulations. (Right top) The unfolded energy distribution of iron. (Right bottom) Systematic uncertainty depending on the initial spectral index in the unfolding procedure compared to an index of -2.60 .

\section{Preliminary Results}

Figure 7 shows the preliminary energy spectra of neon, magnesium, silicon and iron up to 100 $\mathrm{TeV}$ derived from CALET observations. The spectra were plotted above $20 \mathrm{GV}$ in order to avoid the effects of geomagnetic rigidity cutoffs. Each spectrum was calculated using the following equation:

$$
\Phi(E)=\frac{N(E)}{S \Omega \varepsilon(E) T \Delta E}
$$


where $\Phi(E)$ is the differential flux, $N(E)$ is the number of events in the unfolded energy bin, $S \Omega$ is the geometry factor, $T$ is live-time, $\Delta E$ is the width of the energy bin and $\varepsilon(E)$ is the combined efficiency of the trigger and track reconstructions. These spectra are comparable with previous observations by HEAO-3-C2 [18], ATIC-2 [19], TRACER [20-22] and CREAM-II [23]. We note that the event selection we have applied here is very simple and preliminary compared to what can eventually be achieved with CALET. Further studies are now underway to provide a more detailed analysis of these spectra.

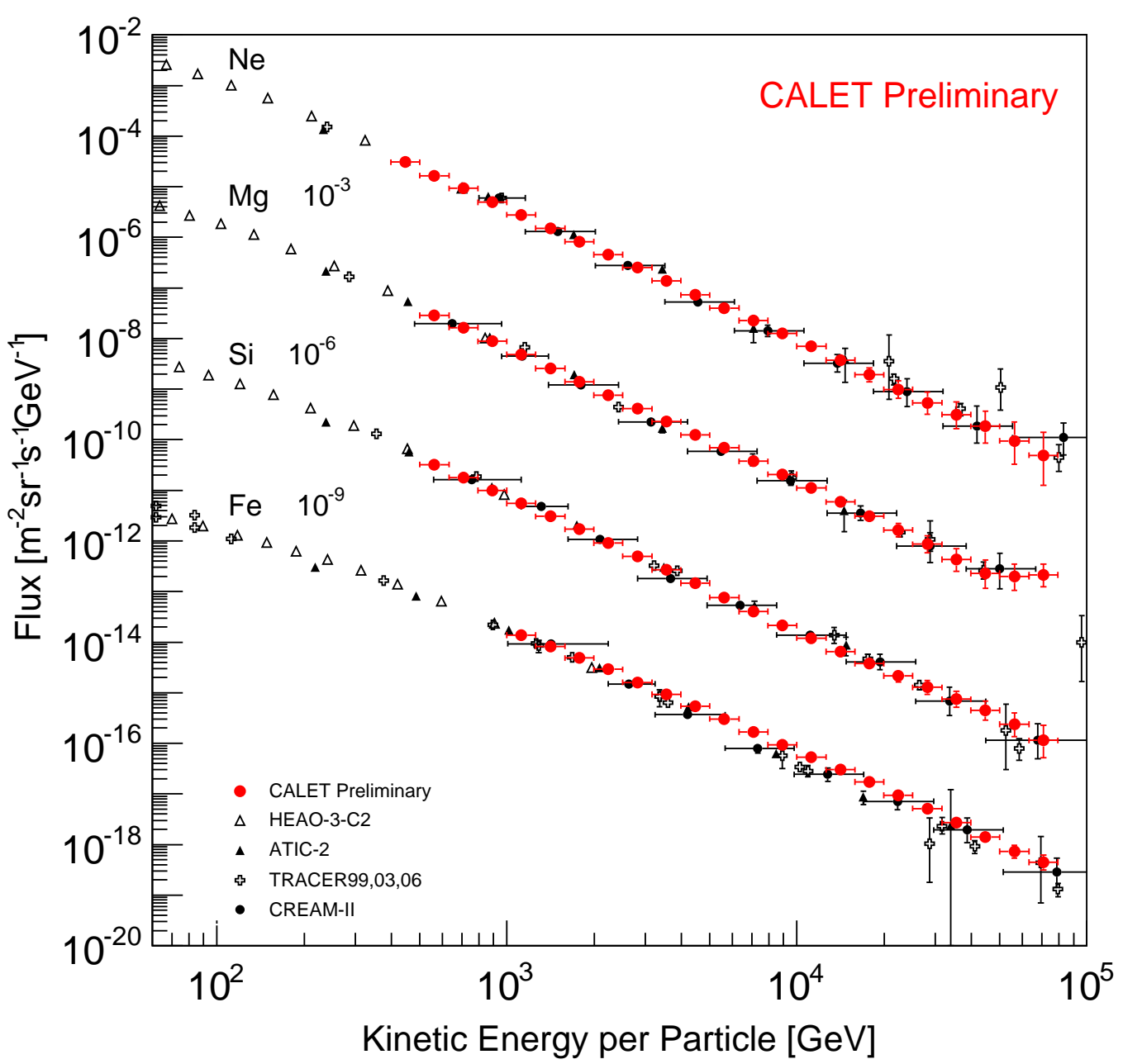

Figure 7: Preliminary flux measured by CALET as a function of kinetic energy per particle for neon, magnesium, silicon and iron cosmic-ray nuclei compared with HEAO-3-C2 [18], ATIC2 [19], TRACER [20-22] and CREAM-II [23]. Only statistical errors are shown.

\section{Summary}

The ability of CALET to measure heavy cosmic-ray nuclei has been successfully demonstrated, and preliminary energy spectra have been derived for the primary cosmic ray elements up to $100 \mathrm{TeV}$ using data from the first 18 months of operation. Detector responses were well studied 
with the latest data, and the efficiency of each analysis procedure was estimated from Monte Carlo simulations in which those responses were effectively implemented. The effects of finite energy resolution and charge resolution on the flux calculation were included in the energy unfolding procedure based on the simulations. The derived spectra prove the excellent capability of CALET to measure heavy ions with high statistics in a wide energy range. Further studies an increased data set will reveal detailed spectral features, which may be a key to solve questions about cosmic-ray acceleration and propagation in our galaxy.

\section{Acknowledgments}

This work was supported in part by a JSPS Grand-in-Aid for Scientific Research (S) (No.25220708), and by the joint research program of the Institute for Cosmic Ray Research (ICRR), University of Tokyo. We thank the NASA Center for Climate Simulation for use of the ADAPT computing system. Author (YA) was supported by JSPS Overseas Research Fellowships.

\section{References}

[1] O. Adriani et al., Science 332, 69 (2011)

[2] M. Aguilar et al., Phys. Rev. Lett. 114, 171103 (2015)

[3] H. S. Ahn et al., Astrophys. J. Lett. 714, L89 (2010)

[4] Y. Akaike et al., Proc. of 33rd ICRC, 0726 (2013)

[5] G. Bigongiari et al., Proc. of 34th ICRC, 592 (2015)

[6] P. S. Marrocchesi et al., Proc. of 33rd ICRC, 0362 (2013)

[7] Y. Akaike et al., Proc. of 34th ICRC, 613 (2015)

[8] Y. Asaoka et al., Proc. of 35th ICRC, this conference (2017)

[9] P. S. Marrocchesi et al., Proc. of 35th ICRC, this conference (2017)

[10] B. Rauch et al., Proc. of 35th ICRC, this conference (2017)

[11] Y. Asaoka et al., Proc. of 34th ICRC, 603 (2015)

[12] Y. Asaoka et al., Astropart. Phys. 91, 1 (2017)

[13] G. Tarlé, S. P. Ahlen, B. G. Cartwright, Astrophys. J. 230, 607 (1979)

[14] G. D’Agostini, Nucl. Instrum. Meth. A 362, 487 (1995)

[15] T. Adye, arXiv:1105.1160

[16] K. Kasahara et al., Proc. of 24th ICRC 1, 399 (1995)

[17] S. Roesler et al., Proc. of Monte Carlo 71, 23 (2003)

[18] J. J. Engelmann et al., A\&A 233, 96 (1990)

[19] A. D. Panov et al., Boll. Russian Acad. Sci. 73, 564 (2009)

[20] F. Gahbauer et al., Astrophys. J. 607, 333 (2004)

[21] M. Ave et al., Astrophys. J. 678, 262 (2008)

[22] A. Obermeier et al., Astrophys. J. 742, 11 (2011)

[23] H. S. Ahn et al., Astrophys. J. 707, 593 (2009) 